\title{
A NOTE ON HADAMARD PRODUCTS OF UNIVALENT FUNCTIONS
}

\section{DAOUD BSHOUTY}

\begin{abstract}
An example is constructed to show that a modified Hadamard product
\end{abstract} of two normalized univalent functions with real coefficients may not be univalent.

Let $S$ denote the class of all functions $f(z)=z+c_{2} z^{2}+\cdots$, analytic and univalent in the unit disk. Given two functions in $S, f_{1}(z)=z+\sum_{n=2}^{\infty} a_{n} z^{n}$ and $f_{2}(z)=z+\sum_{n=2}^{\infty} b_{n} z^{n}$, we define their modified Hadamard product by

$$
\left(f_{1} * f_{2}\right)(z)=z+\sum_{n=2}^{\infty} \frac{a_{n} b_{n}}{n} z^{n}
$$

Let $S_{R}$ be the set of functions in $S$ with real coefficients. In [1] Krzyz questions whether this modified Hadamard product of two functions in $S_{R}$ is in $S_{R}$. The following argument leads to a counterexample. It depends on a weak version of a theorem of Jenkins (see [2, p. 120, Corollary 4.8 and Example 4.5]).

TheOREM. Let $g(z)=z+\sum_{n=2}^{\infty} \alpha_{n} z^{n}$ be in $S_{R}$, and $0<\lambda<2$. If

$$
\alpha_{2}=\lambda(1+\log (2 / \lambda)) \equiv x(\lambda) \text {, }
$$

then

$$
\alpha_{3}<1+\frac{1}{4} \lambda^{2}+\lambda^{2}\left(\frac{1}{2}+\log (2 / \lambda)\right)^{2} \equiv Y(\lambda)=y(x) .
$$

For every choice of $x$, there exists $g_{x}(z)$ in $S_{R}$ for which equality holds in (2).

In fact, given $0<x_{1}<2$, then $h=g_{x_{1}} * g_{x_{2}}$ is noi in $S_{R}$ for $x_{2}$ sufficiently close to 2 .

Note that $h(z)=z+x_{1} x_{2} z^{2} / 2+y\left(x_{1}\right) y\left(x_{2}\right) z^{3} / 3+\cdots$, and if it were in $S_{R}$, then

$$
y\left(x_{1}\right) y\left(x_{2}\right) / 3<y\left(x_{1} x_{2} / 2\right)
$$

Fix $x_{1}$, and put $x_{2}=2-r$, for $0<r<2$, then

$$
\frac{y\left(x_{1}\right)}{3}\left(y(2)-r y^{\prime}(2)+o(r)\right) \leqslant y\left(x_{1}\right)-\frac{r x_{1}}{2} y^{\prime}\left(x_{1}\right)+o(r) .
$$

Received by the editors August 29, 1979 and, in revised form, November 6, 1979.

AMS (MOS) subject classifications (1970). Primary 30A32. 
$y(2)=3$ and from (1) and (2), $y^{\prime}(x)=2 \lambda \log (2 / \lambda)$. We remain with

$$
r \frac{x_{1}}{2} y^{\prime}\left(x_{1}\right)+o(r)<0
$$

which leads to a contradiction for small values of $r$.

\section{REFERENCES}

1. J. M. Anderson, K. F. Barth and D. A. Brannan, Research problems in complex analysis, Bull. London Math. Soc. 9 (1977), 129-162.

2. Ch. Pommerenke, Univalent functions, Vanderhock and Ruprecht, Göttingen, 1975.

Department of Mathematics, Techinion, Israel. Institute of Technology, Hafa, Israkl 